\title{
Place Names of the
}

Avalon Peninsula of the

Island of

Newfoundland 


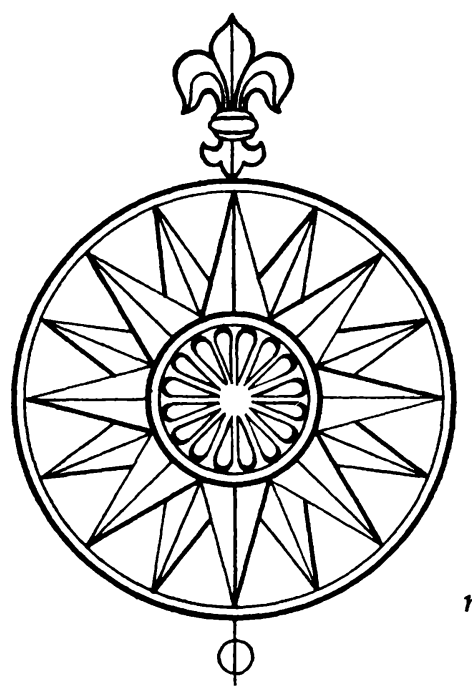

magnifiquement surchargé de noms - PROUST

Published for

Memorial University of Newfoundland by University of Toronto Press 
Place Names of the Avalon Peninsula of the Island of Newfoundland

E.R.SEARY 
MEMORIAL UNIVERSITY SERIES

1 Christmas Mumming in Newfoundland: Essays in Anthropology, Folklore, and History

Edited by HERBERT HALPERT and G.M. STORY

2 Place Names of the Avalon Peninsula of the Island of Newfoundland

BY E.R. SEARY

(C) University of Toronto Press 1971

Toronto and Buffalo

Printed in Canada

Reprinted in 2018

ISBN 0-8020-3243-5

ISBN 978-1-4875-8246-3 (paper)

Microfiche ISBN 0-8020-0068-1

LC 73-151390 
To Gwen 
National Topographic Series,

Newfoundland, with Avalon Peninsula as inset

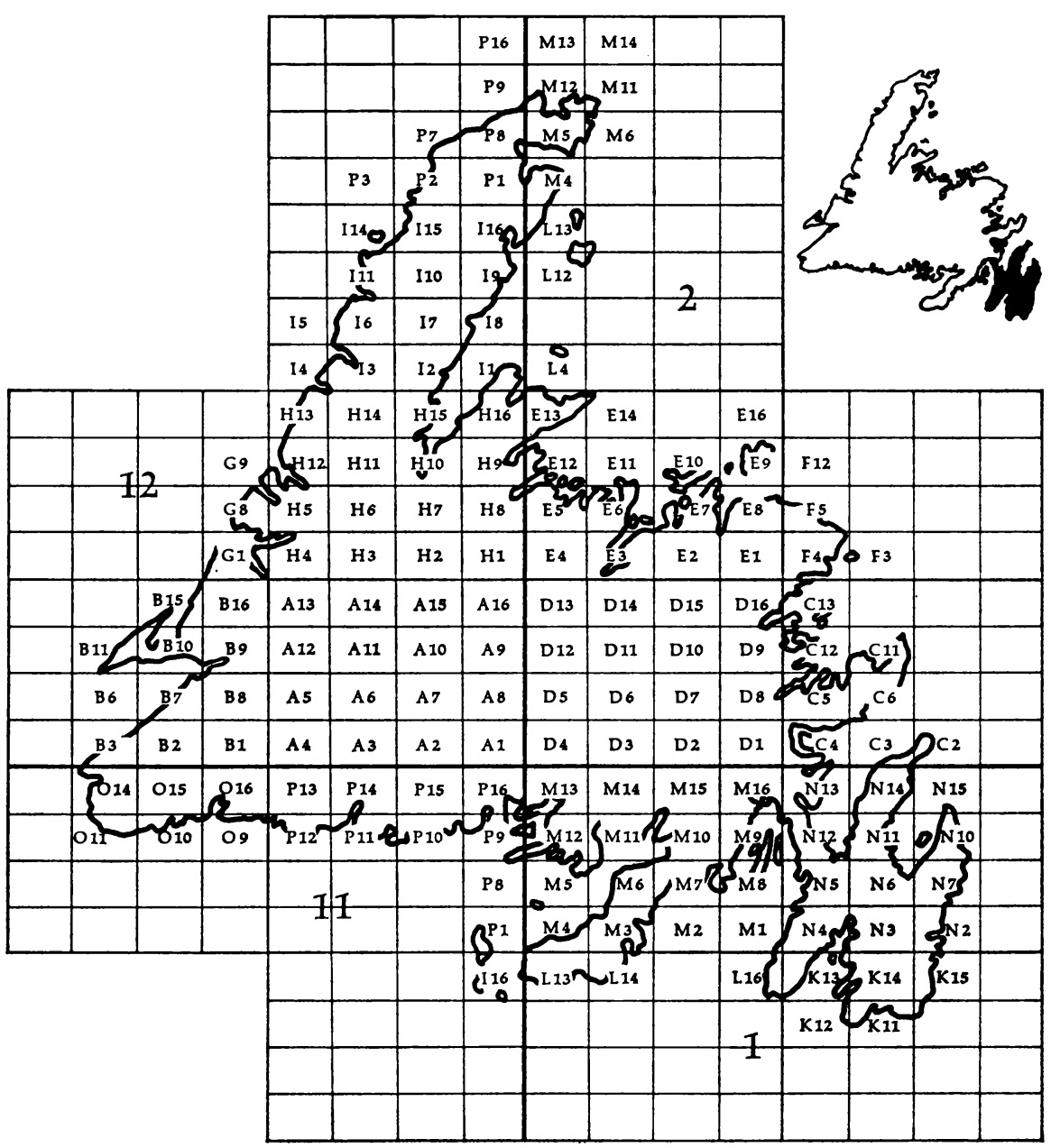




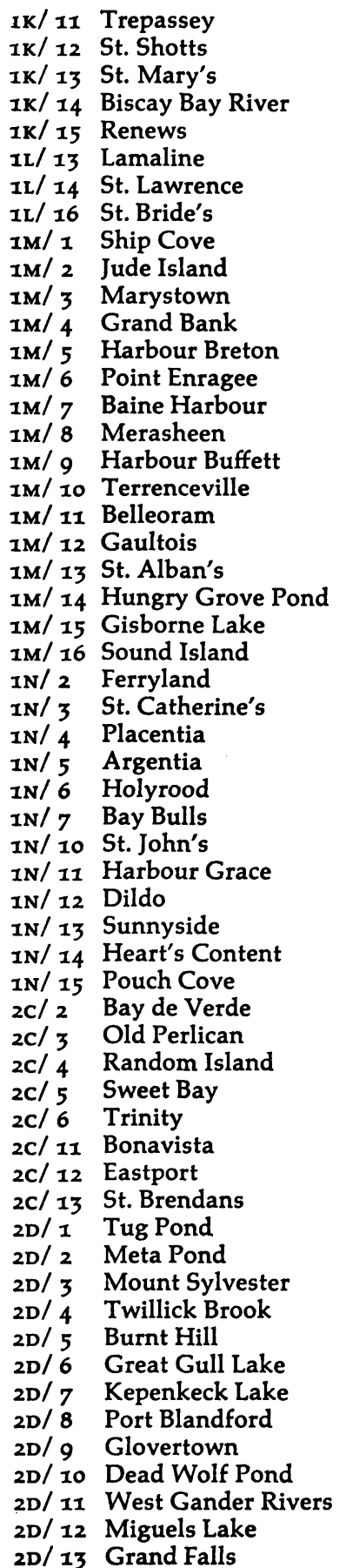

2D/14 Mount Peyton

2D/ 15 Gander

2D/ 16 Gambo

2E/ I Weir's Pond

2E/ 2 Gander River

2E/ 3 Botwood

2E/ 4 Hodges Hill

2E/ 5 Roberts Arm

2E/ 6 Point Leamington

2E/ 7 Comfort Cove

2E/ 8 Carmanville

2E/ 9 Fogo

2E/ 10 Twillingate

2E/ II Exploits

2E/ 12 Little Bay Island

2E/ 13 Nippers Harbour

2E/ 14 Cape St. John

2E/ 16 Bishops Islands

2F/ 3 Cabot Islands

2F/ 4 Wesleyville

2F/ 5 Musgrave Harbour

2F/ 12 Wadham Islands

2L/ 4 Horse Islands

2L/ 12 Grey Islands Harbour

2L/ 13 Groais Island

2M/ 4 St. Julien's

2M/ 5 St. Anthony

2M/ II Quirpon

2M/ 12 Raleigh

2M/ 13 Chateau Point

2M/ 14 Belle Isle

11I/ 16 Ile de St. Pierre

110/ 9 La Poile

110/ 10 Rose Blanche

110/ I1 Port aux Basques

110/ 14 Codroy

110/ 15 Grandys Lake

110/ 16 La Poile River

11P/ I Grande Miquelon

11P/ 8 Pass Island

I1P/ 9 Facheux Bay

11P/ 10 La Hune

11P/ 11 Ramea

11P/ 12 Burgeo

11P/ 13 Peter Snout

11P/ 14 White Bear River

11P/ 15 Dolland Brook

11P/ 16 D'Espoir Brook

12A / I Cold Spring Pond

12A/ 2 Pudops Lake

12A/ 3 Burnt Pond

12A/ 4 King George Iv Lake

12A/ 5 Puddle Pond

12A/ 6 Howley Lake

12A/ 7 Snowshoe Pond

12A/ 8 Great Burnt Lake

12A/ 9 Noel Pauls Brook
12A/ 10 Lake Ambrose

12A/ 11 Star Lake

12A/ 12 Little Grand Lake

12A/ 13 Corner Brook

12A/ 14 Rainy Lake

12A/ 15 Buchans

12A/ 16 Badger

12B/ I Dashwoods Pond

12B/ 2 St. Fintan's

12B/ 3 Little Friars Cove

12B/ 6 Cape St. George

12B/ 7 Flat Bay

12B/ 8 Main Gut

12B/ 9 Harry's River

12B/ 10 Stephenville

12B/ 11 Mainland

12B/ 15 Shag Island

12B/ 16 Serpentine

$12 \mathrm{G} / \mathrm{I}$ Bay of Islands

12G/ 8 Trout River

12G/ 9 Skinner Cove

12H/ 1 Gull Pond

12H/ 2 The Topsails

12H/ 3 Deer Lake

12H/ 4 Pasadena

$12 \mathrm{H} / 5$ Lomond

12H/ 6 Cormack

12H/ 7 Sheffield Lake

12H/ 8 Springdale

12H/ 9 Kings Point

12H/ 10 Hampden

12H/ 11 Silver Mountain

12H/ 12 Gros Morne

$12 \mathrm{H} / 13$ St. Pauls Inlet

12H/ 14 Main River

12H/ 15 Jackson's Arm

12H/ 16 Baie Verte

12I/ 1 Fleur de Lys

12I/ 2 Cat Arm River

121/ 3 Indian Lookout

121/ 4 Portland Creek

12I/ 5 Bellburns

121/ 6 Blue Mountain

121/ 7 Harbour Deep

12I/ 8 Orange Bay

121/ 9 Englee

121/ 10 Torrent River

12I/ 11 Port Saunders

12I/ 14 St. John Island

12I/ 15 Castors River

12I/ 16 Roddickton

12P/ 1 Salmon River

12P/ 2 Brig Bay

12P/ 3 Ferolle Point

$12 \mathrm{P} / 7$ Flower's Cove

12P/ 8 Eddies Cove

12P/ 9 Big Brook 
National Topographic Series, Avalon Peninsula

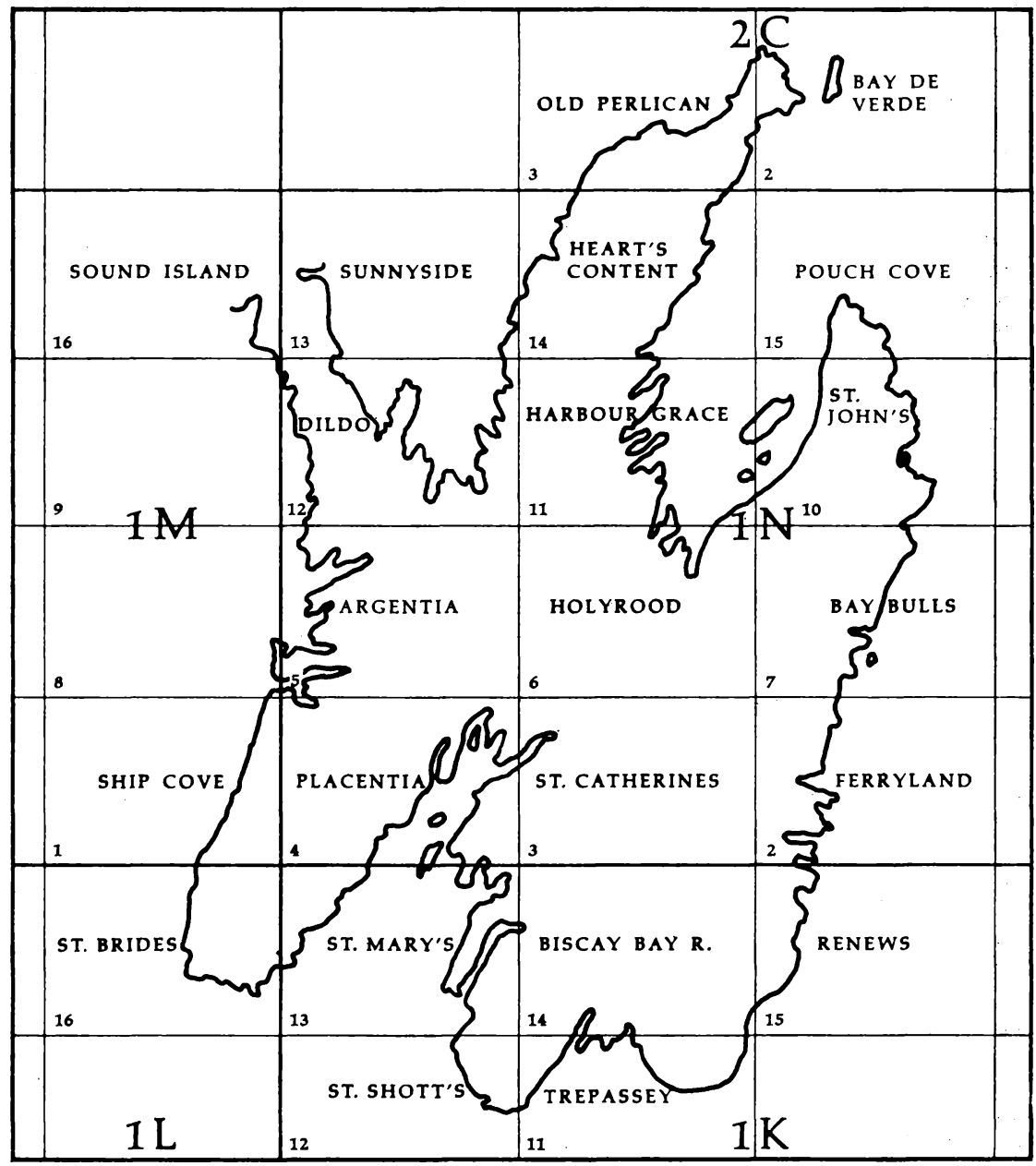

